\title{
Las redes de apoyo familiar: motivación económica y doméstica para allegarse en zonas periféricas de Santiago de Chile.
}

\author{
Family support networks: the economic and domestic motivation to get close to peripheral areas \\ of Santiago de Chile.
}

\section{Valentina Saavedra ${ }^{\mathrm{a}}$ y Paulina Gatica ${ }^{\mathrm{b}}$}

Historial del artículo

Recibido:

18 de junio de 2019

Aceptado:

12 de julio de 2019. a Fundación Vértice, Santiago, Chile. Correo electrónico: vale.saavedra@gmail.com

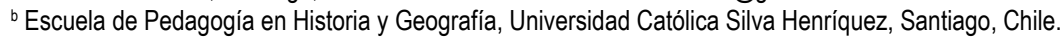

\section{Palabras clave}

Acceso a la vivienda, allegamiento, redes de apoyo familiar

\section{Keywords}

Access to housing, closeness, family support networks

\begin{abstract}
Resumen
La política habitacional implementada en Chile, durante las décadas de 1980 y 1990, fue ampliamente destacada por la capacidad que tuvo de reducir el déficit de vivienda. Sin embargo, las estadísticas actuales demuestran que dicho control, ha perdido efectividad en los últimos años, con un alza del déficit habitacional y el allegamiento, en las comunas que mayor número de vivienda social recibieron durante la época mencionada. La presente investigación propone caracterizar dicha alza y explorar las razones que tiene la población para demandar vivienda en sectores periféricos, lejanos a las zonas de servicios y equipamientos. Para ello se explora desde la teoría, el acceso a la vivienda, el allegamiento y las redes de apoyo familiar. En lo específico, se analizaron dos villas ubicadas en la comuna de Puente Alto en Santiago de Chile, bajo un enfoque metodológico mixto que consideró la utilización de técnicas cuantitativas y cualitativas como análisis de datos estadísticos y entrevistas semiestructuradas. Los resultados de la investigación plantean que son las redes de apoyo las que sustentan el interés de la población por mantenerse en las comunas periféricas, ya que ahí se encuentran sus redes familiares y vecinales, las cuales se constituyen como redes de subsistencia económica y doméstica para hogares, sobretodo, emergentes.
\end{abstract}

\section{Abstract}

Chile, during the 1980's and 1990's, was widely highlighted by its ability to reduce the housing deficit. However, current statistics show that this control has lost its effectiveness in recent years, with a rise in the housing deficit and allegation, in the communes that received the largest number of social housing during the aforementioned period. The present investigation proposes to characterize this rise and explore the reasons that the population has to demand housing in peripheral sectors, far from the service and equipment areas. This is explored from the theory, access to housing, closeness and family support networks. Specifically, two villages located in the district of Puente Alto in Santiago of Chile were analyzed, under a mixed methodological approach that considered the use of quantitative and qualitative techniques such as statistical data analysis and semi-structured interviews. The results of the research suggest that it is the support networks that sustain the interest of the population to stay in the peripheral communes since there are their family and neighborhood networks, which are constituted as economic and domestic subsistence networks for households, above all, emerging. 


\section{Introducción}

La política habitacional chilena se basa, principalmente, en un sistema de subsidios de vivienda enfocados a diferentes segmentos de la población de acuerdo a sus ingresos económicos familiares (Casgrain \& Janoschka, 2013; Inzulza \& Gatica, 2018;). Dicho sistema, proviene de la reforma habitacional que se realizó entre la década de 1970 y 1980 bajo el Régimen Militar, la cual fue consolidada en la década siguiente, con la vuelta a la democracia.

Los resultados de la política habitacional implementada (Ravinet, 2004) fueron destacados a nivel internacional por su capacidad de reducción del déficit habitacional de la época que alcanza el 28,3\%.Sin embargo, desde los estudios sociales y urbanos, ha sido analizada desde un punto de vista crítico ya que significó la construcción de paños homogéneos de vivienda social emplazados en la periferia de la ciudad de Santiago de Chile, con carencia de servicios y equipamiento, y condiciones habitacionales deficitarias (Sabatini \& Arenas, 2000; Sabatini, Cáceres y Cerda, 2001). De esta forma, el crecimiento urbano de comunas como Puente Alto, San Bernardo y Maipú, fue favorecido por la implementación de diferentes programas habitacionales, particularmente entre las décadas de 1980 y 1990.

A pesar de lo anterior, durante los últimos años se ha observado un aumento del déficit habitacional (Cámara Chilena de la Construcción [CChC], 2019), precisamente en aquellas comunas donde se construyó el mayor número de viviendas de las últimas décadas, y que conforman los sectores más alejados de los centros de servicios y equipamientos.

En dicho escenario, esta investigación propone caracterizar esta nueva demanda habitacional, la cual paradójicamente se concentra en aquellas comunas donde por décadas se ha cuestionado la calidad de vida y servicios que ofrece a su población. Es decir, ¿Cómo se explica que, en los sectores con mayor construcción de vivienda social periférica, sea donde mayor déficit habitacional existe?

Como hipótesis se plantea que la solución habitacional desarrollada durante la década de 1990 no logró asegurar condiciones de movilidad social, generando dependencia de las redes de apoyo para el desarrollo familiar. De manera que el gran número de unidades habitacionales construidas en comunas como Puente Alto, San Bernardo y Maipú, conlleva un aumento de allegamiento interno por necesidad de apoyo con redes de cuidados y ahorro lo cual limita el acceso a una vivienda propia.
Los casos de estudio que se analizan en esta investigación corresponden a las villas El Refugio I y El Labrador, construidas en el marco de programas de vivienda social durante la década de 1990. Ambas villas se ubican en Puente Alto, comuna localizada en el extremo sur de la ciudad de Santiago, Región Metropolitana, Chile (Figura 1). El artículo se divide en 4 partes. Primero la introducción y el marco teórico de la investigación con relación al acceso a la vivienda como un derecho, al allegamiento como determinante del déficit habitacional y a las redes de apoyo como parte fundamental para comprender los vínculos entre hogares. Posteriormente, se aborda la metodología, la cual posee un enfoque mixto con métodos cuantitativos y cualitativos para la interpretación de cifras de fuentes oficiales y relatos de los residentes de las villas. Como resultado se evidencia la importancia de las redes de apoyo familiar para la interpretación del déficit habitacional en Chile. Por último, se entregan conclusiones sobre la necesidad de incluir nuevas miradas para comprender la complejidad que abarcan los estudios de vivienda actual.

\section{El acceso a la vivienda como un derecho}

El acceso a la vivienda adecuada es uno de los derechos que establece el Comité de la Organización de las Naciones Unidas de Derechos Sociales, Económicos y Culturales en el año 1966. Se refiere a que todo ser humano debería poder acceder a un espacio de privacidad y familiar, que le entregue seguridad, paz y dignidad. Este derecho promueve la obtención de vivienda bajo condiciones no discriminatorias y en igualdad de condiciones. Además, constituye un paso para el acceso a otros derechos humanos, como la salud, trabajo o la educación (Oficina del Alto Comisionado de Derechos Humanos, 2010). El acceso a la vivienda debe contemplar más allá de un techo, de hecho, se debe abordar el acceso a los recursos necesarios para conseguir una vivienda, por lo que pueden existir diferentes factores de discriminación que generan desigualdad en su asequibilidad, por ejemplo, factores económicos como acceso a créditos o recursos económicos, legales, de emplazamiento o incluso mediados por procesos de participación ciudadana (Centro de Derechos Humanos Universidad Diego Portales, 2016).

Chile, a pesar de suscribir al pacto de los derechos humanos de la Organización de Naciones Unidas [ONU], no establece en su Constitución de la República el derecho a la vivienda. Sin embargo, su acceso es un tema que se ha abordado largamente a través de las políticas habitacionales desde principios del siglo XX y que durante la década de 1980 tuvo respuesta a través de la instalación del programa de subsidios habitacionales. Lo anterior, refleja el rol que 

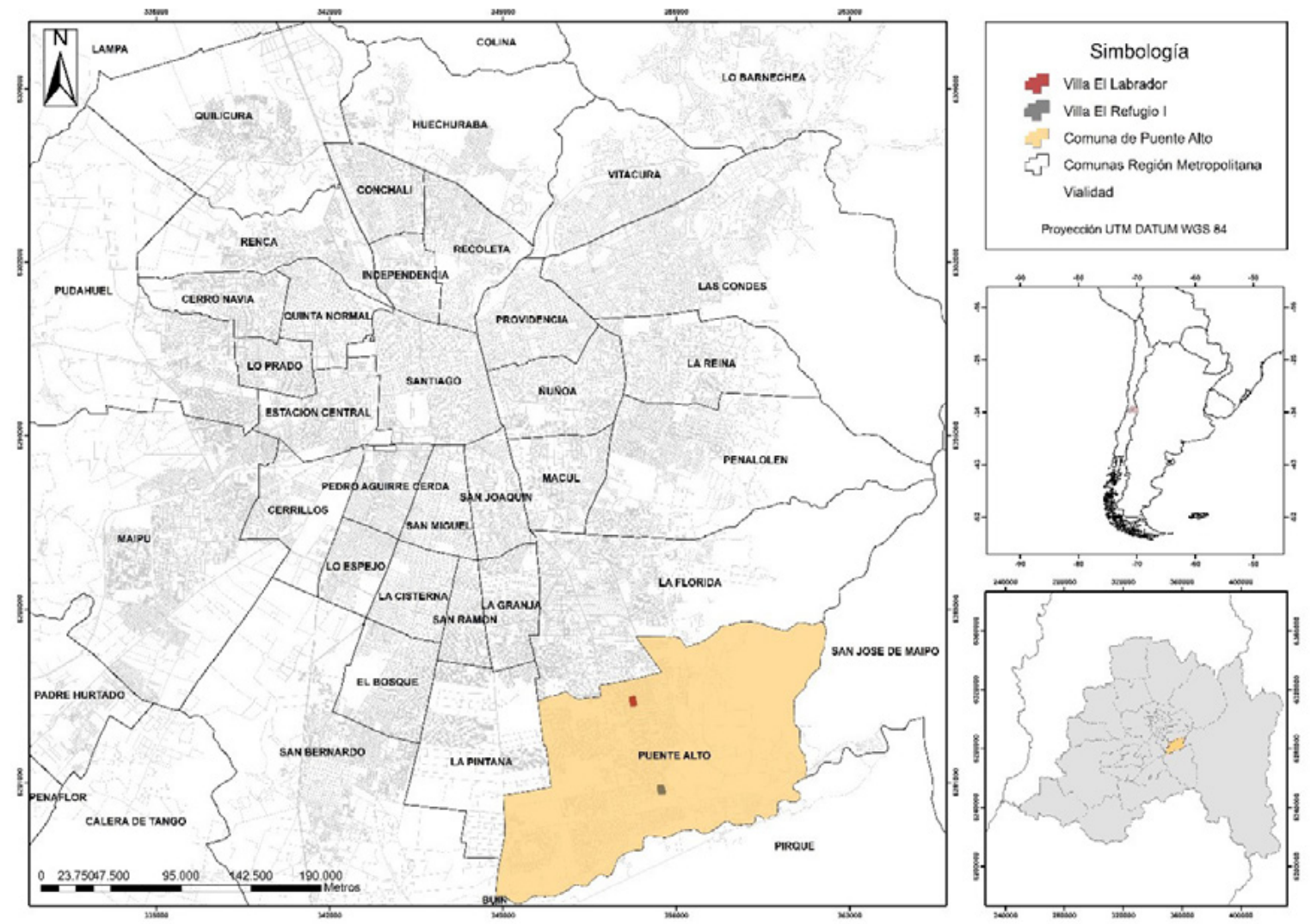

Figura 1. Localización del área de estudio. Fuente: Elaboración propia (2019).

el Estado debe cumplir según establece la Constitución, es decir, que este debe dar solución a aquellos sectores o esferas que el mercado no tiene capacidad de resolver. Por ello, los subsidios van dirigidos a sectores de medios y bajos recursos. De esta manera, la forma de acceder a la vivienda en Chile es distinta según el sector socioeconómico al que pertenece cada sujeto, donde quienes acceden a través de subsidios a la vivienda, requieren de ahorro previo y un crédito aprobado para poder hacerlo (Ministerio de Vivienda y Urbanismo [MINVU], 2019).

\section{El allegamiento como forma de habitar}

Las categorizaciones y dificultades que viven algunos sectores de la sociedad para acceder a la vivienda generan diferentes estrategias de respuesta de los hogares en la búsqueda de una residencia. Una de las principales es el allegamiento. Para CELADE (1996), el allegamiento corresponde a la estrategia utilizada por los hogares y núcleos familiares para solucionar la falta de vivienda, compartiendo una vivienda con otro hogar o núcleo. El allegamiento externo se presenta cuando existe más de un hogar en la vivienda y poseen presupuestos independientes. El allegamiento interno se vincula a la constatación de un núcleo al interior del hogar, el cual se constituye en el núcleo secundario o núcleo allegado. Estos núcleos secundarios son una o más familias que no han logrado aún su autonomía económica y dependen del presupuesto de alimentación y de la vivienda del hogar que los acoge. Esta investigación se centrará, en términos de análisis, en el allegamiento interno, el cual se manifiesta en hogares con familias donde coexisten más de un núcleo familiar, como hijos que formaron un hogar y continúan viviendo con sus padres. De acuerdo a Prieto (2001) el allegamiento interno puede responder a la falta de autonomía económica como a la necesidad de establecer estrategias de sobrevivencia ligadas a problemas de vulnerabilidad para residir de forma autónoma como la seguridad o la necesidad de cuidados, entre otras causas. Esto último genera que el allegamiento sea funcional y que los allegados no sean capaces de demandar una vivienda hasta que logren superar sus condiciones de vulnerabilidad.

El allegamiento se puede analizar desde diferentes enfoques (DESUC, 2017). El allegamiento como estrategia racional de sobrevivencia está vinculado a la generación de cohabitación como una estrategia de maximización de recursos (Araos, 2008), el allegamiento como producto de la demografía, 
asociado a los cambios demográficos de la población y como una forma histórica de estructuración familiar (Arriagada y Moreno, 2008), el allegamiento desde la teoría del parentesco destacando la importancia de los vínculos filiales y la generación de proximidad residencial (Araos, 2016), y por último, el allegamiento desde la localización, relacionado a la preferencia de los hogares por vivir más cerca del centro de la ciudad (Tapia, Araos y Salinas, 2012). A partir de lo anterior, para esta investigación resulta fundamental el análisis del allegamiento como estrategia racional de sobrevivencia (Araos, 2008), como producto de la demografía (Arriagada y Moreno, 2008) y desde la teoría de parentesco (Araos, 2016).

En muchos casos la relación entre allegamiento y hacinamiento es estrecha, no obstante, no todos los hogares allegados viven en condición de hacinamiento. El hacinamiento en Chile es la razón entre el número de personas residentes en la vivienda y el número de dormitorios de la misma, considerando piezas de uso exclusivo o uso múltiple. La medición contempla las categorías: sin hacinamiento, medio y crítico (Ministerio de Desarrollo Social, 2018).

El hacinamiento ha sido estudiado desde diversos enfoques en el mundo y en América Latina (Atkinson 2003; Di Pasquale, 2017; Marcús, 2017; Molina del Villar, 2018). Una de las perspectivas teóricas que se ha abordado, es la perspectiva demográfica, la cual, de acuerdo a Clark, Deurloo \& Dieleman (2010), posee un enfoque vinculado al ciclo de vida de los hogares, demostrando que la necesidad de un espacio habitacional constituye un componente importante en las decisiones familiares ante situaciones que involucran la creación o disolución de hogares, o la generación de un cambio del tamaño del hogar.

Asimismo, el hacinamiento es una variable de alta sensibilidad frente a las modificaciones en los niveles de ingresos económicos asociados a cambios en la escala social, por ende, este fenómeno remite a una compleja interacción entre las características internas de los hogares y condiciones contextuales externas al hogar como la disponibilidad y precio de una vivienda (García, LópezColás y Módenes Cabrerizo, 2018).

De este modo el hacinamiento muchas veces se puede presentar en hogares allegados que no cuentan con capacidad de consumo en el mercado inmobiliario, impidiendo su movilidad residencial y la mejora de su estándar de vida, los cuales pasan a formar parte de los núcleos allegados hacinados que son medidos en el cálculo del déficit habitacional.

\section{Las redes de apoyo familiar}

El allegamiento interno es una de las principales expresiones socio espaciales del resguardo de las redes de apoyo familiar. Las redes de apoyo forman parte de la estructura de relaciones de las personas, que construyen principalmente a través de su vida cotidiana. Como plantea Wellman \& Wortley (1990), las redes de apoyo son de un grado más allá de la red comunitaria o los vínculos que puedan generar las personas con su entorno, ya que consideran una relación que no es unidireccional.

Las redes de apoyo social se dividen entre redes formales e informales, según el tipo de relación (Aranda y Pando, 2013). Las redes de apoyo formales o también denominadas secundarias, son aquellas que se fundan en una estructura y roles establecidos, que requieren una preparación adecuada e instituciones de respaldo, privadas, públicas o comunitarias (Sánchez, 2000). Mientras que las redes de apoyo informales también conocidas como primarias, son aquellas conformadas por la familia, amistades o entorno comunitario y están principalmente fundadas en un componente afectivo, que estimula la socialización y permite satisfacer necesidades básicas (Méndez y Cruz, 2008). Lemos \& Fernández (como se citó en Aranda y Pando, 2013) describen las redes de apoyo informales como círculos concéntricos donde la familia nuclear es el más pequeño y que en conjunto con las amistades, son los círculos internos, mientas los compañeros de trabajo y otros serían parte de círculos externos.

Según el análisis del concepto de Aranda y Pando (2013), las redes de apoyo son motivadas por necesidades emocionales y económicas, generando verdaderas transacciones de respaldo, que tienen como preferencia el apoyo familiar. Esta preferencia, generalmente aparece como solución ante la ausencia de redes institucionales que resuelvan necesidades o carencias, de manera que se ven cruzadas por decisiones de sostenibilidad material y supervivencia familiar (Méndez y Cruz, 2008).

Bonet y Martí (2006) establecen que, así como la vulneración está relacionada con la carencia de vínculos y redes de apoyo, la existencia de estos, en cambio, constituyen un factor fundamental en el análisis de sectores excluidos, pues son variables que pueden disminuir condiciones de desigualdad y vulnerabilidad social y económica. Ducci (1999), plantea que, en Chile, las redes de apoyo familiar cobran especial relevancia, pues debido a la cultura la relación familiar, incluso más allá del núcleo más principal, es cercana y sostenida en el tiempo. 


\section{Materiales y métodos}

Para llevar a cabo esta investigación, se utilizó una metodología con enfoque mixto, mediante métodos y técnicas cuantitativas y cualitativas orientadas a corroborar la hipótesis planteada al inicio de la investigación. Con el propósito de caracterizar el área de estudio en términos demográficos y de vivienda, se analizaron datos censales de población y vivienda de los años 1982, 1992, 2002 y 2017. En el caso específico de las villas de estudio se analizaron sus estadísticas demográficas a través del procesamiento de micro datos censales en Redatam7. Además, se utilizaron datos de la encuesta CASEN 2017, para graficar la evolución del déficit habitacional. Para la generación de material cartográfico se utilizó el software ArcGIS 10.6.

Para el análisis cualitativo se aplicaron cinco entrevistas semiestructuradas a pobladores de las villas El Labrador y El Refugio 1, de manera de entregar un acercamiento a la realidad que experimentan personas en situación o relación con el allegamiento y sus razones. Por ello, se entrevistaron a cuatro mujeres y un hombre, adultos y residentes de las villas, quienes cumplían diferentes perfiles: un dirigente vecinal, dos mujeres jefas de familia, una en situación de allegamiento y hacinamiento y una en situación de allegamiento sin hacinamiento actual, y dos mujeres madres de familias biparentales, una que decidió su localización por cercanía familiar y una que decidió localización lejos de las redes familiares.

El análisis de las entrevistas se realizó a través de la técnica de análisis de contenido con el objetivo de corroborar la dependencia de los hogares con sus respectivas redes de apoyo.

\section{Elección del caso de estudio}

El área de estudio de la presente investigación se encuentra en la comuna de Puente Alto, ubicada en la periferia sur del Gran Santiago, Chile. Puente Alto posee una población de 568.106 habitantes (INE, 2017) y junto a San Bernardo y Maipú, constituye una de las comunas con mayor déficit habitacional a la fecha, con 22.441 unidades (CChC, 2019).

Para efectos de esta investigación se seleccionaron 2 villas de la comuna de Puente Alto: El Refugio 1 y El Labrador (Figura 1). Ambas villas fueron entregadas a través del Programa de Vivienda Básica (PVB), creado en el año 1982 como respuesta del Estado al déficit habitacional que en esa época alcanzaba el $28,3 \%$ de familias de escasos recursos las cuales integraban la población de "marginalidad habitacional" (Saavedra, 2017). En la actualidad el PVB ya no existe como tal, debido a una serie de modificaciones que sufrieron los programas de vivienda social durante la década de 1990 y 2000 . No obstante, constituye uno de los programas habitacionales más influyentes de la historia urbana de Santiago de Chile.

\section{Resultados}

De acuerdo a los resultados de la Encuesta CASEN 2017, el déficit habitacional cuantitativo es un indicador que mide el número de viviendas requeridas para dar alojamiento a hogares allegados, núcleos secundarios hacinados y para reemplazar viviendas irrecuperables (Ministerio de Desarrollo Social, 2018). En Chile, alcanza las 497.615 unidades de Vivienda (Ministerio de Desarrollo Social, 2018), número similar a las 497.012 viviendas en déficit en el año 1998, lo cual significa un retroceso de 20 años en términos de déficit habitacional (Figura 2).



Figura 2. Composición del déficit habitacional cuantitativo por requerimiento 1996-2017 en Chile. Fuente: Elaboración propia a partir de CASEN, 2017. 


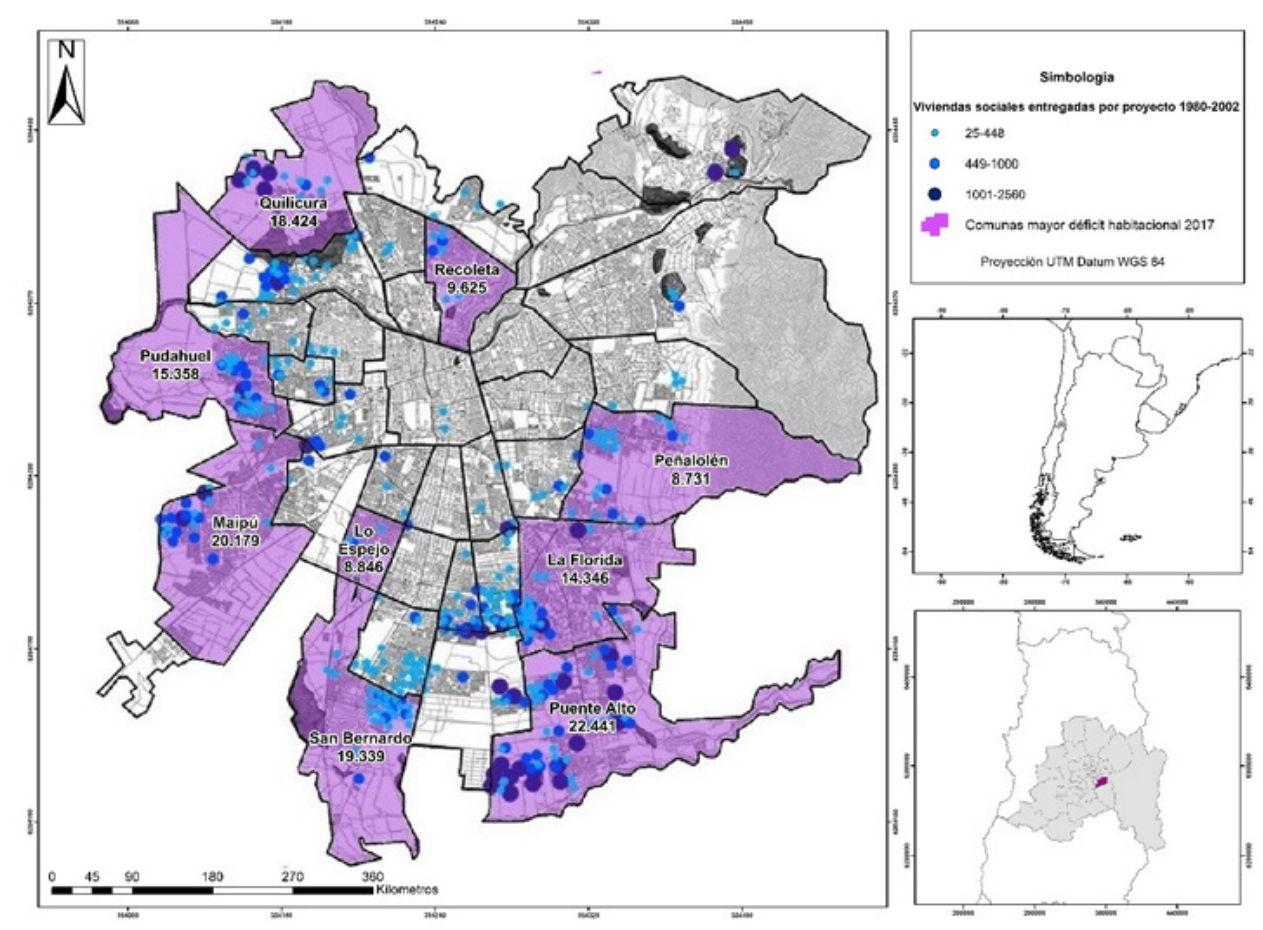

Figura 3. Localización del área de estudio. Fuente: Elaboración propia (2019).

Tal como se expresa en la Figura 3, la principal razón en el aumento del déficit habitacional actual se encuentra en el considerable aumento que tuvieron los hogares allegados, entre el año 2015 y 2017, lo cual significó un aumento del 37\% a nivel nacional. De acuerdo a los informes redactados a partir de la publicación de datos de la encuesta CASEN 2017 por instituciones de gobierno (Ministerio de Desarrollo Social, 2018) y privadas (CChC, 2019), los motivos tras la decisión de allegarse tienen relación a la preferencia por mantener una buena localización que les permita a los hogares acceder a servicios y equipamientos, $\mathrm{y}$ también, al aumento sostenido de precio de las viviendas en los últimos años. De acuerdo a la Cámara Chilena de la Construcción (2019), durante los últimos 10 años, el precio de las viviendas ha aumentado en más de $100 \%$ en la Región Metropolitana, mientras que los salarios han crecido un $36 \%$. Ello implica que, si hace 15 años un hogar necesitaba 8,8 años de ingresos para comprar una vivienda, ahora necesita de 16 años.

Si bien, el acceder o mantener una buena localización es una de las razones más poderosas que tienen los hogares para allegarse, y por ende, buscar estrategias de co-residencia (Urrutia y Cáceres, 2019), son las comunas periféricas las que hoy concentran el mayor déficit habitacional por unidad de vivienda (Figura 3), las mismas que fueron las mayores receptoras de vivienda social durante las décadas de 1980 al 2000, como es el caso de la comuna de Puente Alto, que recibió 26.131 viviendas sociales en el periodo en cuestión y que hoy presenta un déficit habitacional de 22.441 unidades de vivienda.

Abufhele (2019), señala que, a principios de la década de 1990, el allegamiento respondía a una "demanda social contenida" (p.62). Para satisfacer dicha demanda, el primer gobierno de la Concertación, implementó medidas y programas destinados a aumentar la construcción de viviendas, reducir el déficit habitacional y prevenir la toma de terrenos. Dichas medidas y programas reforzaron las soluciones habitacionales propuestas durante la dictadura, periodo en que se diseñó y ejecutó el Programa de Vivienda Básico (PVB) como respuesta del Estado al déficit habitacional dirigido a los sectores de menores ingresos. De acuerdo a Abramo (2014), la producción de las ciudades modernas en América Latina resulta del funcionamiento de tres lógicas de coordinación social: la del mercado, la del Estado y la de la necesidad. En este caso, las implicancias territoriales de los programas de vivienda subsidiadas por el Estado en la ciudad de Santiago de Chile, se traducen en un impulso más para el crecimiento centrífugo de la ciudad (Sousa, 2010) potenciando la expansión urbana hacia la periferia. 
- Vivienda $\quad$ Población

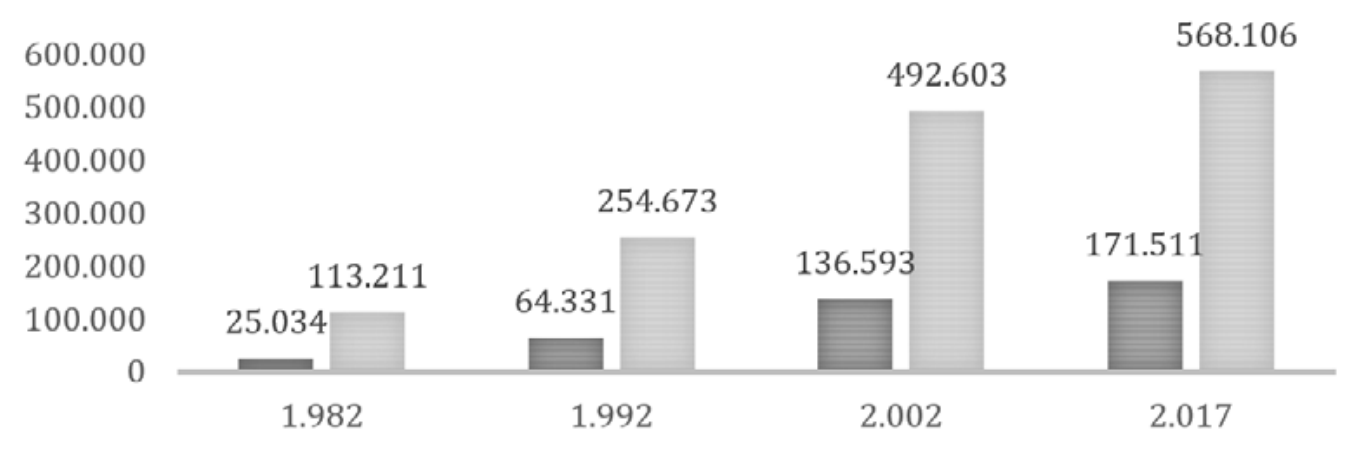

Figura 4. Población y vivienda de la comuna de Puente Alto, Santiago, Chile. 1982-2017. Fuente: Elaboración propia a partir de censos 1982. 1992, 2002 y 2017. INE, 2018.

La comuna de Puente Alto, desde 1982, ha experimentado un crecimiento sostenido en materia de población y vivienda (Figura 4). Según los datos del censo 2017, actualmente posee una población de 568.106 habitantes y 171.511 viviendas, erigiéndose como la comuna más poblada de Santiago y del país (INE, 2018). Durante el periodo intercensal de 1982 a 1992, la comuna obtuvo una tasa de crecimiento medio anual de un $8,1 \%$, mientras que en el periodo de 1992 a 2002, tuvo un crecimiento medio anual de 6,6\%. El crecimiento explosivo de la población, en esta época, coincide con el periodo en que se entregó el mayor número de subsidios de vivienda en la comuna. Entre 1980 y 2002 se construyeron 26.131 viviendas sociales en la comuna de Puente Alto, convirtiéndose en la comuna con mayor recepción de vivienda social del Gran Santiago (Tabla 1), por sobre La Florida y San Bernardo (Tapia, 2011).

\section{El caso de las villas El Labrador y El Refugio 1}

Dos de las villas construidas durante esta época en Puente Alto, son las villas El Labrador y El Refugio 1, emplazadas en el sector norte y sur de la comuna (Figura 5), relativamente cercanas a la línea 4 del Metro de Santiago.

En términos población, y de acuerdo a los resultados del Censo 2017 (Tabla 2), la villa El Labrador posee una población de 2.207 habitantes, los cuales son mayoritariamente mujeres y del grupo etario correspondiente a población económicamente activa (que se encuentra entre los 15 y 64 años). En el caso de la villa El Refugio 1, está compuesta por 2.897 habitantes, los cuales al igual que el caso de la villa El Labrador, corresponde mayoritariamente a mujeres y del grupo etario entre los 15-64 años.

Tabla 1

Las diez comunas con mayor recepción de vivienda social del Gran Santiago, entre 1980-2002.

\begin{tabular}{lll}
\hline Posición & Comuna & Cantidad de viviendas sociales \\
\hline 1 & Puente Alto & 26.131 \\
\hline 2 & La Florida & 23.506 \\
\hline 3 & San Bernardo & 22.927 \\
\hline 4 & La Pintana & 15.396 \\
\hline 5 & Peñalolén & 13.204 \\
\hline 7 & Pudahuel & 13.190 \\
\hline 8 & Maipú & 11.582 \\
\hline 9 & El Bosque & 10.304 \\
\hline 10 & Renca & 9.116 \\
\hline
\end{tabular}

Fuente: Elaboración propia a partir de Tapia (2011). 
Tabla 2

Caracterización demográfica de las Villas El Labrador y El Refugio 1, Puente Alto, 2017.

\begin{tabular}{ccccccccccccc}
\hline \multicolumn{1}{c|}{ Datos de Población } & & \multicolumn{3}{c|}{ Datos de vivienda } \\
\hline Villa & $\begin{array}{c}\text { Total de } \\
\text { personas }\end{array}$ & $\begin{array}{c}\text { Total de } \\
\text { Hombres }\end{array}$ & $\begin{array}{c}\text { Total de } \\
\text { Mujeres }\end{array}$ & $\mathbf{0 - 5}$ años & $\mathbf{6 - 1 4 \text { años }}$ & $\mathbf{1 5 - 6 4 \text { años }}$ & $\mathbf{6 5}$ y más años & Viviendas & $\begin{array}{c}\text { Viviendas } \\
\text { censadas }\end{array}$ & $\begin{array}{c}\text { Número de } \\
\text { hogares }\end{array}$ \\
\hline El Labrador & 2.207 & 1.070 & 1.137 & 175 & 245 & 1.631 & 156 & 674 & 645 & 652 \\
\hline El Refugio 1 & 2.897 & 1.399 & 1.498 & 293 & 415 & 2.044 & 145 & 825 & 806 & 814 \\
\hline
\end{tabular}

Fuente: Elaboración propia a partir del censo 2017

Respecto a los datos de vivienda (Tabla 2), a partir del número de viviendas efectivamente censadas, se observa que en ambos casos la cantidad de hogares es superior a las viviendas. Incluso es probable que esta cifra sea mayor en la realidad, dada la confusión que muchas familias censadas tienen de la definición de hogar para efectos del censo y la diferencia entre las viviendas y las viviendas censadas.

Villa EI Labrador. La Villa El Labrador, se encuentra ubicada en el sector norte de Puente Alto y comenzó a poblarse 1990. Los beneficiados de estas viviendas postularon de forma individual, por lo cual, en la mayoría de los casos, no existían redes vecinales previas. Se encuentra ubicada al norte de la comuna de Puente Alto, y está delimitada por las calles El Inquilino por el norte, El Labrador por el sur,
Nonato Coo al oeste y Los Ñandues al oriente (Figura 5). En la Villa El Labrador, predomina como tipología de vivienda, las casas de dos pisos (Figura 6). La villa está compuesta por 674 casas pareadas de $45 \mathrm{~m}^{2}$ de agrupación continua, que se organiza en pasajes y se emplazan en sitios de $80,9 \mathrm{~m}^{2}$ con antejardín.

Villa El Refugio I. La Villa El Refugio 1, en tanto, está emplazada en el sector sur de Puente Alto y comenzó a poblarse 1994. Los beneficiados fueron comités de vivienda que se organizaron a fines de la década de 1980 para acceder a una vivienda social al contrario de la Villa El Labrador donde la postulación fue individual. Se encuentra ubicada al sur de la comuna de Puente Alto, y está delimitada por las calles Profesor Alcaíno por el norte, Francisco Allen por el sur, Calle Uno por el este


Figura 5. Localización Villa El Labrador y El Refugio 1. Fuente: Elaboración propia (2018). 
y Tomé por el oeste (Figura 5). Además, en su límite sur colinda con los terrenos de la Cárcel de Puente Alto. En el caso de la Villa El Refugio I, la tipología de vivienda predominante son los blocks de departamento y las casas de dos pisos (Figura 6). Está compuesta por 21 pares de blocks de tres pisos, de escaleras cruzadas, que suman 504 departamentos de $45 \mathrm{~m}^{2}$ los cuales comparten un patio interior. También existen 214 casas de dos pisos de 19,62 $\mathrm{m}^{2}$, de agrupación continua, distribuidas en pasajes.

\section{Motivaciones domésticas y económicas tras el allegamiento y la permanencia}

Para poder indagar sobre las razones que provoca el allegamiento y la búsqueda de vivienda propia en la actualidad, o hace algunos años en barrios periféricos, se entrevistó a diferentes residentes de la población El Labrador y El Refugio 1 de Puente Alto, de manera de detectar las motivaciones de su permanencia en el sector, las que se basan principalmente en las redes de apoyo que asegura la relación familiar, que reemplaza labores de cuidados cuando no hay acceso público o privado a servicios de esa índole y entrega seguridad o confianza. Así lo menciona la entrevistada $\mathrm{N}^{\circ} 1$, cuando se consulta por las motivaciones para quedarse en la villa, quien vive actualmente con su marido y sus tres hijos en la Villa El Refugio 1 de Puente Alto.

"A mí me salió mi departamento para un sector que no fue de mi agrado y por el bienestar de mi hijo mayor, decidimos venirnos para acá porque estaba cerca de mi mami, porque yo trabajaba y necesitábamos que a Javier lo viera gente de confianza, entonces nos vinimos a arrendar acá a la villa y yo arrendé mi departamento. Y bueno ahí se dieron un sinfín de cosas, mi mamá se compró al final esa casa. Es de

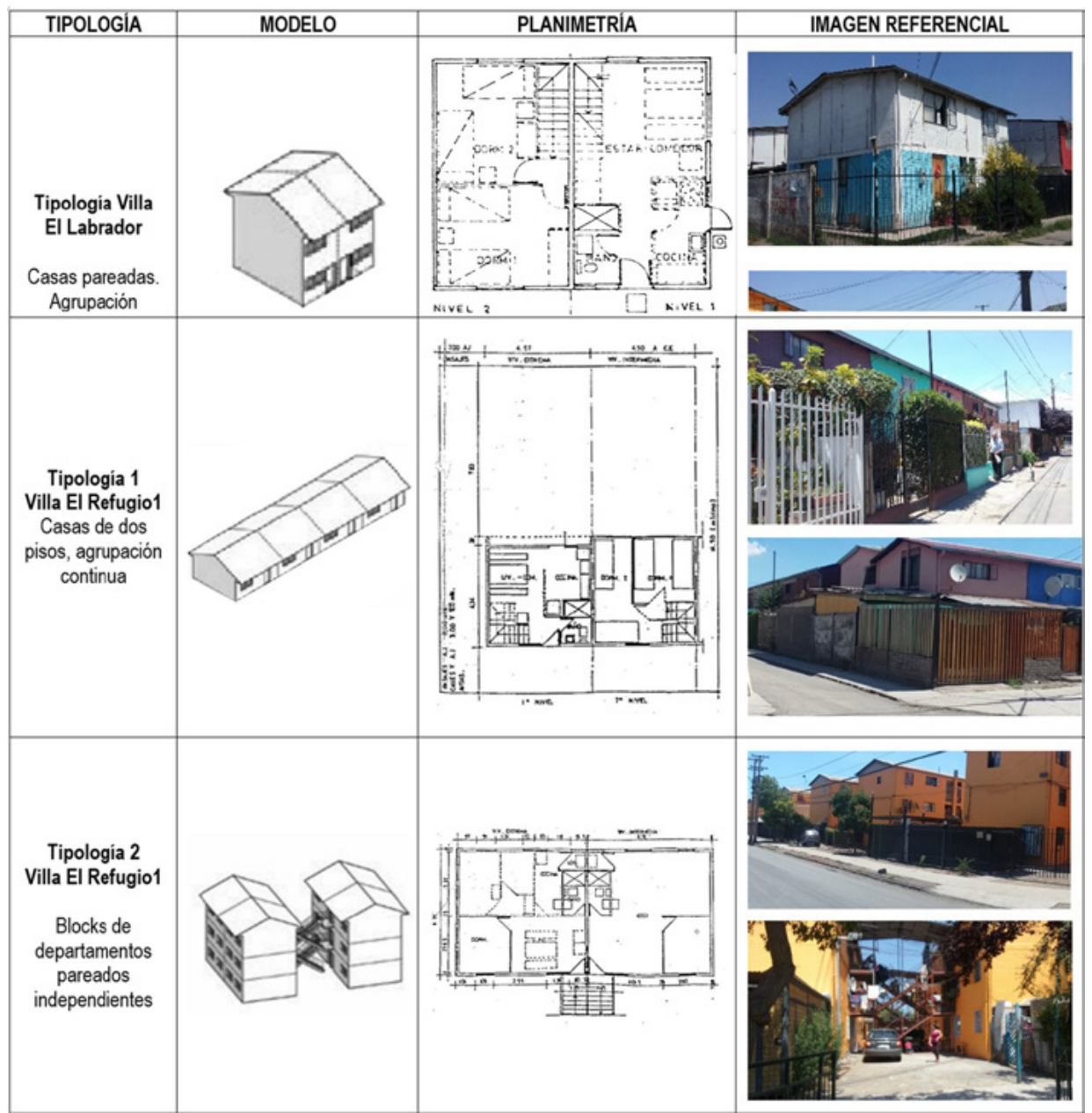

Figura 6. Tipología de vivienda Villa El Labrador y El Refugio1. Fuente: Fuente: Saavedra (2017). 
mi madre esa casa, mi mamá tiene dos casas acá y yo tengo mi departamento" (Entrevistada $\mathrm{N}^{\circ} 1$, Villa El Refugio 1, 10/12/2017).

De esta forma, a pesar que una nueva vivienda en otro sector de la ciudad generaba otros atributos de calidad de vida como la seguridad, no resolvió problemas asociados a la vida cotidiana, como el abastecimiento y cuidado de la familia, en particular de personas dependientes como el caso de los niños o a las personas mayores.

La elección de dónde residir, se ha transformado en un punto importante para los hogares, sobre todo aquellos que están recién comenzando. En casos como el reflejado por la entrevistada $\mathrm{N}^{\circ} 1$, a la hora de decidir si optar por una vivienda propia en otra comuna, lejana de su familia o mantenerse cerca de las redes de apoyo, hay habitantes que optan por lo segundo, postergando la independencia de su familia nuclear a la espera de una solución habitacional cercana, principalmente por las necesidades que resuelve el vivir cerca de sus redes de apoyo familiar.

En la misma línea la entrevistada $\mathrm{N}^{\circ} 2$ relata que gracias a la permanencia de su familia nuclear en la casa de su madre, ella ha podido generar ingresos propios para mantener a su hija, ya que de lo contrario, no tendría posibilidades de trabajar o sus aspiraciones laborales debiesen reducirse, así como los ingresos familiares.

"Es que yo cuando estoy trabajando, todo eso mi mamá lo hace, mi mamá se dedica a que (mi hija) tome desayuno, después le da su almuerzo, la viste para el colegio, porque mi hija va en la tarde, le da almuerzo y después se va al colegio. Después yo llego una hora, media hora más tarde que ella." (Entrevistada N², Villa El Refugio 1, 13/12/2017)

Esta situación se ha transformado en algo recurrente, en especial en los sectores de menores recursos de la población, pues la red de apoyo cobra una relevancia de nivel de subsistencia emocional y económica en la vida diaria, ya que entrega sustento que permite ausentarse de la vivienda para trabajar, realizar compras o recreación, por haber distribuido entre más personas las labores domésticas y de cuidados.

Incluso, optar por tener vivienda propia lejos de las redes de apoyo familiar implica asumir las consecuencias de esa decisión, donde generalmente son las mujeres de la familia las que reciben el mayor impacto de ello. El principal requerimiento que pueden resolver las redes familiares, tienen relación a trabajos domésticos y de cuidados, comúnmente asignados o asumidos por las mujeres, lo mismo con el apoyo emocional que estas resuelven. Esta situación es señalada por la entrevistada $\mathrm{N}^{\circ} 3$, quien está casada y es madre de tres hijos, y expresa el sacrificio personal y económico que significó la lejanía de su núcleo familiar extenso al irse a vivir a la villa El Labrador, renunciando a sus estudios y la posibilidad de trabajar fuera de la casa durante la infancia.

"Los mismos dramas, chuta mejor me quedo en la casa porque no tengo quién me cuide a los hijos, mis papás viven fuera, no tienes a alguien a quién sacrificar. Tengo enfermeras, tituladas... ¡uf! mucha gente" (Entrevistada N³, Villa El Labrador, 10/12/2017).

Este extracto es parte de la descripción que nos hizo sobre los sacrificios que significó vivir lejos de las redes familiares, lo que implicó no poder terminar sus estudios de abogada y perder la posibilidad de mayores ingresos para su familia nuclear, ya que dependió de ella el cuidado total de sus tres hijos, cuestión -que según su relato- es común en el barrio donde vive.

\section{Experiencia tras el allegamiento}

A través de las entrevistas también se abordó cómo había sido la experiencia de las personas de los barrios estudiados viviendo como allegadas en casa de sus familias.

Las consecuencias de vivir en situación de allegamiento, generalmente distan de una buena convivencia o calidad de vida, lo que se acrecienta cuando quienes conviven, provienen de la misma familia en términos extensos, ya que se mezclan roles de responsabilidades y ascendencias familiares entre familias nucleares. Por lo tanto, la decisión de mantenerse en un área cercana a las redes de apoyo, conlleva asumir las implicancias de ello, que van desde la falta de espacio por la cantidad de personas que viven en el mismo espacio, compartiendo servicios higiénicos, espacios comunes y en ocasiones dormitorios. Convivir con tensiones asociadas al uso del equipamiento y espacios de la vivienda, la falta de la privacidad e intimidad y tensione por la distribución de gastos y compras.

"Siempre hemos sido nosotros cuatro, después cuando yo tenía como ocho años se vino a vivir mi otro hermano por parte de papá para acá y ahí igual era incomodo...teníamos una pieza y ahí teníamos que dormir los tres. Entonces igual era incómodo. Después llegó mi primo, como mis papás son gendarme lo ayudaron a postular a gendarmería, entonces él vivió once años con nosotros acá, pero él vivió ya cuando 
la casa estaba ampliada, y él dormía en la misma pieza que mi hermano, pero él vivió once años con nosotros y se compró casa aquí al frente (Entrevistada №4, Villa El Labrador, 13/12/2017).

El allegamiento de la familia extensa y la permanencia en la vivienda de los hijos de la familia nuclear cuando se convierten en padres, es una situación que se repite en diferentes casos en las villas de estudio. El régimen de convivencia, en ese sentido, es la tónica del diario vivir de las familias más jóvenes de las villas, aunque no siempre resulta exitosa por diferentes motivos relacionados a la crianza de los hijos y la convivencia con el resto de las personas que residen en la vivienda.

“Ah, y el papá de él (hijo), que duró dos años aquí... no po, en el 2005 no. Mi hijo nació el 2011. Entonces ahí llegó mi primo y vivió 11 años. Después, en el 2011, fue cuando llegó el papá de mi hijo, y ahí éramos 6. Y ahí a finales del 2011 llegó mi hijo, y ahí éramos 7, y éramos pelea todos los días, el caos en la casa, el baño, porque aquí hay dos baños, pero lamentablemente si yo uso el baño acá abajo, y alguien usa el baño arriba, le salía el agua helada po, entonces eran peleas, que apúrate, que no había continuación, que justo yo iba a entrar al baño y entrai tú, era un ambiente bien poco acogedor" (Entrevistada $\mathrm{N}^{\circ} 4$, Villa El Labrador, 13/12/2017).

Una experiencia similar comentó la entrevistada $\mathrm{N}^{\circ} 4$, quien vive en la villa El Refugio I, es madre soltera, vive con seis de sus ocho hermanos, tres hijos de sus hermanos, su hijo y su madre. Conformando cuatro familias nucleares en la misma vivienda, todas allegadas debido al apoyo que entrega la familia para el cuidado de los hijos y optimizar los gastos cotidianos de cada una.

"El espacio en sí, igual se hace chico, intentamos de hacer, pero sabis que nunca están todos, siempre es en la noche que están todos, ya algunos se van a acostar y algunos están de pie, pero nunca están, así como todos juntos. Cuando están todos juntos, no se puede caminar y los gritos y los llantos, ya que es mucho, no mal. Prácticamente en la semana no hay gente, porque algunos estudian y mis hermanas también trabajan, entonces no se ve mucha gente" (Entrevistada $N^{\circ}$ 2, Villa El Refugio I, 13/12/2017).

Una externalidad que genera el allegamiento, tiene relación con los efectos que produce en el barrio, el sobre-uso del espacio y que acrecienta la urgencia de respuesta a la demanda habitacional del sector. Estas consecuencias se pueden observar en la carencia de espacios de uso colectivo para los vecinos, en ocasiones la saturación de las calles y pasajes con autos estacionados o ampliaciones por necesidad de espacio, que afecta el espacio público.

"Hay algunos pasajes que tienen ese tema organizado, como jardines y árboles, pero hay otros pasajes que no porque, porque sucede eso, porque resulta que como los pasajes son tan angostos la mayoría con el tiempo ha ido adquiriendo sus vehículos, el espacio de las casas, muchos han construido, han hecho ampliaciones en sus viviendas por lo tanto no les ha quedado patio y estacionan sus vehículos afuera...Ustedes van a ver después en las viviendas, si quieren visitar una vivienda, la van a ver. Mucha gente ha tenido que ampliarse porque las casas son chicas y con el tiempo ha quedado poco patio, pero hay varios pasajes que se preocupan del tema de tener arbolitos, pastito y se mantiene y se organizan para mantener eso" (Entrevistado N ${ }^{\circ}$, Villa El Labrador, 13/12/2017).

Como se puede observar a partir de los extractos de las entrevistas realizadas, las personas admiten lo dificultoso que puede significar la vida como allegado y la pérdida de sentido de pertenencia con la vivienda donde residen, así como los efectos que implica para el espacio comunitario y público de los barrios, sin embargo, tampoco avizoran alternativas que resuelvan las condiciones económicas, materiales, de cuidados, tiempos y emocionales, como sí lo hace la cercanía con la familia nuclear o extensa, por lo que la espera e insistencia en conseguir un subsidio para una vivienda localizada en las proximidades de sus redes de apoyo, cobra relevancia y merece la pena por sobre la búsqueda de ubicación en los centros de servicios y trabajo. La decisión que están tomando las familias en proceso de constitución se condice con el aumento de demanda de vivienda en zonas periféricas, como es el caso de la comuna de Puente Alto, donde son los y las hijas de las familias receptoras de los subsidios asignados durante la década de 1980-1990, quienes pretenden mantener su localización generando un aumento del déficit por su residencia allegada en casa de sus familiares.

\section{Discusión}

La demanda habitacional actual se encuentra concentrada en comunas que décadas atrás fueron las que más recibieron viviendas a través de subsidios habitacionales del Programa de Vivienda Básica, esto se ha convertido en un punto de atención, precisamente, porque deben en gran medida su crecimiento poblacional y territorial a la entrega de 
viviendas a través de subsidios del Estado. A la vez, son comunas periféricas, que generalmente son vinculadas a la carencia de servicios, conectividad y equipamiento, como lo es la comuna de Puente Alto, caso de estudio de esta investigación, la cual continúa experimentando un alza sostenida en materia de población y vivienda y también de déficit habitacional.

En cuanto a la relación entre la tipología de la vivienda y el modo de habitar de los hogares de las villas abordadas en este estudio, se observa que las familias han tenido que modificar la estructura tradicional para brindar un techo al resto de los integrantes de la familia allegados. Si bien los datos censales del último censo, no muestran altas cifras de hogares allegados, si es un aspecto latente que es abordado en casi todas las entrevistas realizadas. En consecuencia, los resultados permiten observar que los factores que determinan preferencia de localización, no se restringen exclusivamente a las facilidades clásicamente analizadas desde los estudios urbanos, como la cercanía a centros laborales o la adquisición de servicios, pues existen ciertas actividades que inciden en la vida cotidiana que van adquiriendo un mayor valor, que son los cuidados y las labores domésticas de la familia, lo que permite caracterizar de mejor manera las redes de apoyo que se construyen, con la familia y amigos cercanos y los niveles de interdependencia que se genera entre ellos.

En ambos casos, el grupo etario predominante pertenece al rango etario que constituye la población económicamente activa en Chile. En ese sentido, las entrevistas reflejan que las familias que constituyen los hogares son población trabajadora que requiere de redes de apoyo relacionadas al cuidado y el trabajo doméstico para poder desarrollarse laboralmente. La mayoría de las veces este rol recae en mujeres, las cuales, en las dos áreas de estudio, son mayoría poblacionalmente.

\section{Conclusiones}

En esta investigación se demuestra que la generación y existencia de redes de apoyo constituyen una razón fundamental para muchas familias a la hora de tomar la decisión de establecerse en una vivienda. De esta forma, las redes de apoyo incluso se sobreponen al deseo de optar a una mejor localización dentro de la ciudad. Lo anterior ha sido demostrado a través de las entrevistas realizadas a diferentes residentes de las villas El Refugio 1 y El Labrador, donde las redes de apoyo han sido fundamentales para cubrir necesidades cotidianas, disminuyendo costos de vida, debido a la distribución de gastos domésticos. De la misma manera con necesidades vinculadas al cuidado y el trabajo doméstico, lo que principalmente para familias jóvenes ha significado la posibilidad de generar ingresos propios estables con la búsqueda de un trabajo formal, fuera del hogar y con un horario que requiere de externalizar el cuidado de sus hijos. Aquello es una razón fundamental a la hora de preferir la cercanía con la red de apoyo, ya que, ante contextos con carencias de servicios, desligarse -en parte- de los cuidados de las personas dependientes, requiere que otro miembro de la familia o círculo cercano, se haga cargo. Estas, son razones de peso ante la elección del lugar de residencia de los hogares, independiente que este se ubique en un sector periférico que no goza de las virtudes del equipamiento, infraestructura y servicios que ofrece el centro y pericentro de la ciudad.

De este modo se comprueba la hipótesis: se ha generado una dependencia de las redes de apoyo para el desarrollo familiar, algo que las políticas urbanas desarrolladas desde la década de 1980 no lograron prever. En ese sentido, las soluciones habitacionales implementadas no lograron asegurar condiciones de movilidad social para la descendencia de los beneficiados por los subsidios de vivienda en comunas periféricas como Puente Alto, lo cual se ve agravado por los altos precios del mercado inmobiliario actual lo que provoca que muchas familias vean frustrados sus intentos de independencia, buscando solución habitacional en las cercanías de su familia. Lo que en definitiva concentra un alto déficit habitacional por allegamiento.

Aunque la investigación que aquí se presentó, no resulta metodológicamente representativa de la dinámica habitacional nacional, sí logra demostrar con información fehaciente la necesidad de incluir otras variables para poder analizar y comprender las diferentes dimensiones que implica el déficit habitacional. Las redes de apoyo constituyen un factor determinante en la elección de residencia, y su estudio, debe incorporarse en los estudios urbanos y territoriales, entendiendo que, a pesar de los cambios sociales y culturales de las familias, son absolutamente necesarias en un contexto urbano y social desigual.

Con lo expuesto anteriormente, una línea de desarrollo que permitiría ahondar en el análisis, se relaciona con el valor que toman las redes primarias para cubrir los cuidados en sectores de la población de menores ingresos, que son fundamentales para construir procesos de independencia económica para las familias jóvenes y cómo aquello, podría cuestionar la forma de categorización tradicionalmente utilizada en las investigaciones sobre la localización. Permitiendo abordar factores que podrían considerar una buena localización, sin ser necesariamente centros productivos, sino de cuidados. 


\section{Referencias}

Abramo, P. (2012). La ciudad com-fusa: mercado y producción de la estructura urbana en las grandes metrópolis latinoamericanas. EURE, 38(114), 35-69. https://dx.doi.org/10.4067/S0250-71612012000200002

Abufhele, V. (2019). La política de la pobreza y el gobierno de los asentamientos informales en Chile. EURE, 45(135), 49-69. https://doi.org/10.4067/s025071612019000200049

Aranda, C., y Pando, M. (2013). Conceptualización del apoyo social y las redes de apoyo social. Revista de Investigación en Psicología, 16(1), 233-245. https:// doi.org/10.15381/rinvp.v16i1.3929

Araos, C. (2008). La tensión entre filiación y conyugalidad en la génesis empírica del allegamiento. Estudio cualitativo comparado entre familias pobres de Santiago de Chile (Tesis de postgrado). Pontificia Universidad Católica de Chile, Santiago, Chile.

Araos, C. (2016). When family lives nearby: Kinship, socioeconomic conditions and residential configurations in Santiago, Chile. Cuadernos ISUC. Working papers series, 1-18.

Arriagada, C., y Moreno, J. C. (2008). El crecimiento de los hogares en Chile. Proyección y análisis de la evolución de los hogares a escala nacional, regional y comunal (2002-2018). Santiago: Ministerio de Vivienda y Urbanismo.

Atkinson, A. (2003). Multidimensional Deprivation: Contrasting Social Welfare and Counting Approaches.

The Journal of Economic Inequality, 1 (1), 51-65. https:// doi.org/10.1023/A:1023903525276

Bonet i Martí, Jordi (2006). La vulnerabilidad relacional: análisis del fenómeno y pautas de intervención. Redes. Revista Hispana Para El Análisis de Redes Sociales, 11(2), 1-17. https://dx.doi.org/10.5565/rev/redes.89

Cámara Chilena de la Construcción [CChC) (2019). Déficit habitacional: un desafío pendiente. Recuperado de https://bit.ly/2WPzYkn

Casgrain, A. y Janoschka, M. (2013). Gentrification and Resistance in Latin American Cities: The Example of Santiago de Chile. Andamios, 10(22), 19-44.
CELADE (1996). Déficit habitacional y datos censales sociodemográficos: una metodología. CEPAL: Santiago.

Centro de Derechos Humanos UDP (2016). Informe anual sobre derechos humanos en Chile 2016. Recuperado de https://bit.ly/2uTd5QH

Clark, W.A.V., Deurloo, M.C., \& Dieleman, F.M. (2000). Housing Consumption and Residential Crowding in U.S. Housing Markets. Journal of Urban Affairs, 22(1), 49-63. https://doi.org/10.1111/0735-2166.00039

DESUC (2017). Estudio factores que influyen en el allegamiento interno y externo. Recuperado de https:// bit.ly/2ui3ys9 _

Di Pasquale, E. A. (2017). Medición de las condiciones habitacionales: una propuesta metodológica para analizar desigualdades inter e intraterritorios urbanos. Cuadernos de Vivienda y Urbanismo, 10(20), 22. https:// doi.org/10.11144/javeriana.cvu10-20.mchp

Ducci, M. (1999). Chile: el lado oscuro de la política de vivienda exitosa. EURE, 23(69), 99-115.

García, D. M., López-Colás, J., y Módenes Cabrerizo, J. A. (2018). Análisis sociodemográfico de las condiciones objetivas y subjetivas del hacinamiento en España. Cuadernos de Geografía: Revista Colombiana de Geografia, 27(1), 195-213. https://doi.org/10.15446/ redg.v27n 1.56990

Inzulza-Contardo, J., \& Gatica-Araya, P. (2018). Subsidiary displacement and empty plots: Dilemmas of original residents and newcomers in the reconstruction of Talca, Chile 2010-2016. Urban Studies, 56(10) 1-18. https:// doi.org/10.1177/0042098018787967

Marcús, J. (2017). "Mi casa es esta pieza": migración femenina y precariedad habitacional en la Ciudad de Buenos Aires. Cuestiones de Sociología, 17, e040. https://doi.org/10.24215/23468904e040

Méndez, E., y Cruz, L. (2008). Redes sociales de apoyo y persona adulta mayor: Costa Rica. Documento preparado para el I Informe del Estado de Situación de la Persona Adulta Mayor en Costa Rica, UCR/ CONAPAM.

Ministerio de Desarrollo Social (2018). Síntesis de resultados CASEN 2017. Recuperado de https://bit.ly/2ObQMhn 
Ministerio de Vivienda y Urbanismo [MINVU] (2019). Beneficios de vivienda construida. Recuperado de https://bit.ly/2Kq5AeS

Molina del Villar, A. (2018). Vivir en la orfandad, pobreza y hacinamiento. Los asilos constitucionalistas y las condiciones de vida y salud de los niños en la ciudad de México, 1915-1918. Estudios de Historia Moderna y Contemporánea de México, 55, 195-242. https://doi. org/10.22201/iih.24485004e.2018.55.64521

Oficina del alto comisionado de derechos humanos de la ONU (2010). El derecho a una vivienda adecuada. Recuperado de https://bit.ly/209AZn9

Prieto, B. (2001). Determinantes de la situación de allegamiento interno en las familias de bajos ingresos (Tesis de postgrado). Pontificia Universidad Católica de Chile, Santiago, Chile.

Ravinet De La Fuente, J. (2004). La Política Habitacional Chilena: alternativas de acceso a la vivienda para las familias más pobres. Revista INVI, 19(50), 130-145.

Saavedra, V. (2017). Desarticulación social y espacial como efectos de la politica habitacional focalizada. Santiago de Chile 1980 a 1997 (Tesis de pregrado). Universidad de Chile, Santiago, Chile.

Sabatini, F., \& Arenas, F. (2000). Entre el Estado y el mercado: resonancias geográficas y sustentabilidad social en Santiago de Chile. EURE, 26(79), 95-113.

Sabatini, F., Cáceres, G., \& Cerda, J. (2001). Segregación residencial en las principales ciudades chilenas: tendencias de las tres últimas décadas y posibles cursos de acción. EURE, 27(82), 21-42. https://doi. org/10.4067/s0250-71612001008200002

Sánchez, C. 2000. Gerontología Social. Editorial Espacio. Buenos Aires, Argentina.

Souza, E. (2010). De la ciudad a la Metrópoli. Una interpretación teórica del fenómeno expansivo ligado a la vivienda a la vulnerabilidad y a la pobreza: El caso del área metropolitana de Monterrey, Nuevo León, México. Revista INVI, 25(69), 19-101. https://doi. org/10.4067/S0718-83582010000200002

Tapia, R. (2011). Vivienda social en Santiago de Chile. Análisis de su comportamiento locacional, periodo
1980-2002. Revista INVI, 26 (73), 105-131. https:// doi.org/10.4067/s0718-83582011000300004

Tapia, R., Araos, C., \& Salinas, C. (2012). Condominios familiares. Una alternativa de solución residencial para familias allegadas en lotes tipo $9 * 18$. En Centro de Políticas Públicas UC (Ed.), Propuestas para Chile (pp. 101-137). Santiago: Ediciones UC.

Urrutia, J. P., y Cáceres, M. (2019). Co-residencia: independencia en la restricción. ARQ (Santiago), 101, 108-119. https://dx.doi.org/10.4067/S071769962019000100108 